\title{
Nanosensing Backed by the Uncertainty Principle
}

\author{
I. Filikhin, A. Karoui, and B. Vlahovic \\ Department of Physics, North Carolina Central University, 1801 Fayetteville Street, Durham, NC 27707, USA
}

Correspondence should be addressed to I. Filikhin; ifilikhin@nccu.edu

Received 4 December 2015; Accepted 5 January 2016

Academic Editor: Wen Zeng

Copyright (C) 2016 I. Filikhin et al. This is an open access article distributed under the Creative Commons Attribution License, which permits unrestricted use, distribution, and reproduction in any medium, provided the original work is properly cited.

\begin{abstract}
Possibility for a novel type of sensors for detecting nanosized substances (e.g., macromolecules or molecule clusters) through their effects on electron tunneling in a double nanoscale semiconductor heterostructure is discussed. We studied spectral distributions of localized/delocalized states of a single electron in a double quantum well (DQW) with relation to slight asymmetry perturbations. The asymmetry was modeled by modification of the dot shape and the confinement potential. Electron energy uncertainty is restricted by the differences between energy levels within the spectra of separated QWs. Hence, we established a direct relationship between the uncertainty of electron localization and the energy uncertainty. We have shown in various instances that a small violation of symmetry drastically affects the electron localization. These phenomena can be utilized to devise new sensing functionalities. The charge transport in such sensors is highly sensitive to minuscule symmetry violation caused by the detected substance. The detection of the electron localization constitutes the sensor signal.
\end{abstract}

\section{Introduction}

A new generation of nanosensors is underway and is expected to revolutionize modern engineering fields, including bioengineering and drug delivery systems in nanomedicine, to name a few. Semiconductor heterostructures, such as quantum dots and rings, are of high interest for the development of these nanosensors, as well as many other predicted nanodevices. Of particular importance is the electron tunneling that occurs between the nanosized elements of such devices. Double quantum systems facilitate the study of tunneling related to barrier penetration effects in double well potentials [1]. When the elements of a quantum system get coupled, the energy barrier splitting of degenerate levels occurs, due to their common wave function. As a result, two nearly degenerate eigenstates are formed, which are a linear combination of the wave functions of the electron in isolated dots. Likewise, electron spectrum of double quantum dots formed by a set of quasidoublets is well described within the one-dimensional formalism given in [1] for double quantum wells. An example of theoretical analysis for double quantum dots has been presented in $[2,3]$. These are supported by experimental studies of different aspects (spin effects, coupling distance, electron-phonon coupling, hybrid nanostructures, etc.) which are tightly connected to, and controlled by, charge tunneling in the DQDs. These aspects are extensively developing; see, for instance, [46]. Recently, we have reported in [7] that single electron localized/delocalized states and the tunneling in DQWs and DQDs are highly sensitive to the violation of reflection symmetry of the dots. It should be noted that this aspect is relevant to quantum dot reality, as fabrication technologies produce dot arrays with imperfect shape and distributions. These imperfections inherently induce a chaotic behavior in the QDs. It has been demonstrated that chaos strongly influences charge transport and other properties of QDs $[8,9]$. The relevance of electron tunneling and chaos in real QD arrays is obvious for next generation nanodevices, for instance, future single molecule nanosensors, quantum computing devices, and advanced solar cells, to name a few.

In the present work we study the spectral distribution of electron localized/delocalized states and tunneling in DQWs. The average coordinate is used to characterize the localization of a single electron. We discuss the case of identical QWs constituting a DQW, as an example, when the uncertainty principle is manifested. Uncertainty of electron localization occurs when the difference of electron energies in the left and right QW is very small; that is the case of almost identical 
QWs. The symmetry violation caused by differences in the geometry and/or the confinement potentials in left and right QWs is thoroughly discussed.

\section{Effective Model for InAs/GaAs Quantum Dots}

We consider quantum dots composed of InGaAs on a GaAs substrate. The fabrication of such kind of quantum dots is reported in $[10,11]$, for example. In practice, QDs have average lateral size and height of 41 and $1.6 \mathrm{~nm}$, respectively, with variations within $23 \%$ and $28 \%$, respectively. Hence, in our model the heterostructured QD dimensions were varied within these limits. The QDs were laterally distributed (two-dimensional array) for minimizing the computational recourses. The underlying quantum problem is modeled utilizing the kp-perturbation single subband approach, which is mathematically formulated by the Schrödinger equation as follows [12]:

$$
\left(\widehat{H}_{\mathbf{k p}}+V_{c}(r)+V_{s}(r)\right) \Psi(r)=E \Psi(r) .
$$

Here $\widehat{H}_{\mathbf{k p}}$ is the single band $\mathbf{k p}$-Hamiltonian operator $\widehat{H}_{\mathbf{k p}}=-\nabla\left(\hbar^{2} / 2 m^{*}\right) \nabla, m^{*}=m^{*}(\mathbf{r})$ is the electron effective mass, which depends on the position of the electron, and $V_{c}(\mathbf{r})$ is the band gap potential, which is null inside the QW, $V_{c}(\mathbf{r})=0$, and constant, equal to $V_{c}$, outside the QW. The value of $V_{c}$ is defined by the conduction band offset for the bulk. The band gap potential for the conduction band is chosen as $V_{c}=0.594 \mathrm{eV}$ [13]. The magnitude of $V_{c}$ is calculated as $V_{c}=k\left(E_{g, 2}-E_{g, 1}\right)$, where $E_{g, 1}$ and $E_{g, 2}$ are the band gaps of QD and the substrate, respectively. The coefficient $k$ can be different for the conduction and valence bands. Here, the dimensionless constant $k$ values are taken from [14]. For the conduction band $k(\mathrm{CB})=0.54$, and for the valence band $k(\mathrm{VB})=0.46$. Using experimental values $E_{g, 1}=0.42 \mathrm{eV}$ and $E_{g, 2}=1.52 \mathrm{eV}, V_{c}=0.594 \mathrm{eV}$ was obtained for the conduction band. The bulk effective masses of InAs and GaAs are $m_{1}^{*}=0.024 m_{0}$ and $m_{2}^{*}=0.067 m_{0}$, respectively [15], where $m_{0}$ is the free electron mass. $V_{s}(\mathbf{r})$ is the effective potential simulating the strain effect; it is attractive and acts inside the QW. The magnitude of the potential can be chosen [12] to reproduce experimental data. In presented work, the magnitude of $V_{s}$ for the conduction band is $0.21 \mathrm{eV}$. With this value, the results of the 8 th band $\mathbf{k p}$-calculations of [16] are well reproduced [17].

\section{Wave Function of Two Level System}

Single electron spectrum of a two-level system is defined as a set of quasidoublets [1]. The one-dimensional wave functions of each quasidoublet can be expressed as follows [1]:

$$
\begin{aligned}
& \Psi_{+}=\cos \left(\frac{\Theta}{2}\right) \psi_{1}+\sin \left(\frac{\Theta}{2}\right) \psi_{2}, \\
& \Psi_{-}=-\sin \left(\frac{\Theta}{2}\right) \psi_{1}+\cos \left(\frac{\Theta}{2}\right) \psi_{2},
\end{aligned}
$$

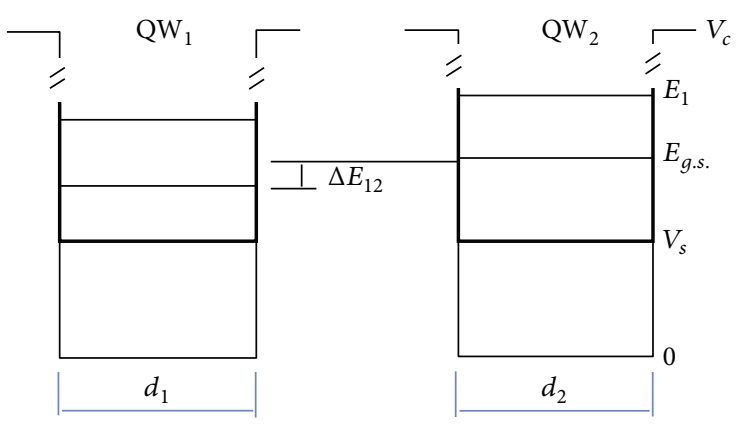

FIGURE 1: Band gap model for one-dimensional DQW. $d_{1}$ and $d_{2}$ are widths of the QWs, $d_{1} \neq d_{2}$. $\Delta E_{12}$ is the difference between homolog energy levels pertaining to left and right QWs, considered separated. The notations are corresponding to the theoretical model described above. $E_{\text {g.s. }}$ is ground state energy of an electron.

where

$$
\tan (\Theta)=\frac{2 W}{\Delta E_{12}} \quad \text { with } 0 \leq \Theta \leq \pi .
$$

Relation (2) shows that the wave function decomposed onto the basis set $\left(\psi_{1}, \psi_{2}\right)$. The parameter $W$ is a coupling coefficient of the quantum system elements. It depends on the wave function overlap for the "unperturbed states" $\psi_{1}$ and $\psi_{2}$ of the left and right quantum dots considered to be separated. $\Delta E_{12}=E_{1}-E_{2}$ is energy difference of separated QWs. It is defined for each energy level in the spectrum. An illustration of the band gap model with the effective potential is presented in Figure 1.

In (2), we neglected the phase shift as it does not play a role in the present case. The energy difference $\Delta E_{12}$ coincides with the quasidoublet splitting $\Delta E=E_{+}-E_{-}$for separated QWs.

To evaluate the electron localization, we analyze the single electron average coordinate $\langle x\rangle$, calculated as $\langle x\rangle_{i}=\langle i|x| i\rangle$ for $i=1,2$, which are associated, respectively, with $\mathrm{QW}_{i}, i=$ 1,2 , considered to be separated. The $x$-coordinate origin is the midpoint of the two QWs. The average coordinate $\langle x\rangle_{+}$ $\left(\langle x\rangle_{-}\right)$of the electron in $\Psi_{+}$state $\left(\Psi_{-}\right)$can be written as

$$
\begin{aligned}
\langle x\rangle_{+}= & -\cos ^{2}\left(\frac{\Theta}{2}\right)\left|\langle x\rangle_{1}\right|+\sin ^{2}\left(\frac{\Theta}{2}\right)\left|\langle x\rangle_{2}\right| \\
& +2 \sin \left(\frac{\Theta}{2}\right) \cos \left(\frac{\Theta}{2}\right)\langle x\rangle_{12}, \\
\langle x\rangle_{-}= & -\sin ^{2}\left(\frac{\Theta}{2}\right)\left|\langle x\rangle_{1}\right|+\cos ^{2}\left(\frac{\Theta}{2}\right)\left|\langle x\rangle_{2}\right| \\
& -2 \sin \left(\frac{\Theta}{2}\right) \cos \left(\frac{\Theta}{2}\right)\langle x\rangle_{12}
\end{aligned}
$$

for corresponding levels of a quasidoublet spectrum (i.e., the DQW spectrum). Due to the dependence on $\Theta$, single electron spectrum is constituted of three parts: delocalized states $(\Theta \approx \pi / 2)$, localized states $(\Theta \approx 0)$, and states with different probability for localizations in left and right QWs. The equation $\Theta=\operatorname{arctg}\left(W / \Delta E_{12}\right)$, derived from 


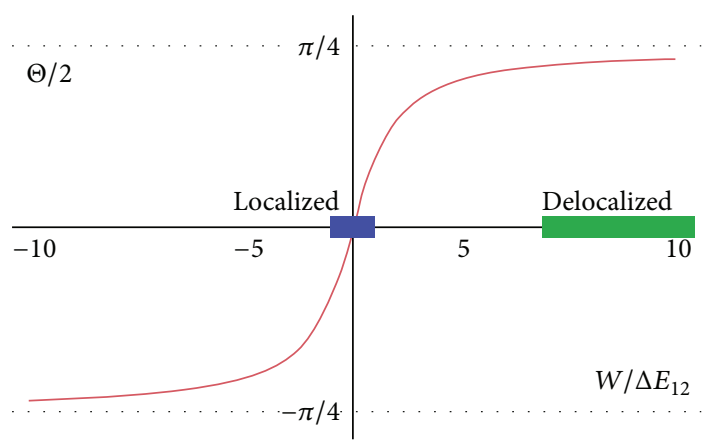

FIGURE 2: Relation between the parameter $\Theta$ and the QW coupling coefficient in a DQW. The ranges for localized and delocalized states of the electron in DQW are shown.

(3), provides the relationship between $\Theta$ parameter and localization/delocalization likelihood of electron in DQW. Figure 2 illustrates that relationship and shows the domain ranges where location and delocalization occur.

According to relation (3), the electron localization in DQW is extremely sensitive to small violations of DQW symmetry, when $\Delta E_{12} \approx 0$. By differentiation of relation (3), one can obtain an estimate of the sensitivity of the terms in (2) to small variations of the energy difference $\Delta E_{12}$ :

$$
\delta\left(\frac{\Theta}{2}\right) \sim \frac{W}{\left(\Delta E_{12}\right)^{2}} \delta\left(\Delta E_{12}\right)
$$

Due to the square in the denominator, small variations of $\Delta E_{12}$ cause large variations of the prefactors in relations (2) and (4). Hence, for weakly coupled QWs the uncertainty of single electron energy is minimal, while the uncertainty for electron localization is large. This fact is in line with the uncertainty principle.

\section{Numerical Results}

4.1. Identical QWs. The experimentally demonstrated circular disc shaped InAs/GaAs quantum dots constitute double quantum wells. We consider in this section identical quantum wells, with equal radii $R_{1}=R_{2}=33 \mathrm{~nm}$. An example of experimental realization of this system is reported in $[10,11]$, where the grown QDs are highly symmetric and free from any lateral elongation.

The electron average coordinate $\langle x\rangle$ in this DQW system has been calculated for each level of the single electron spectrum, according to the above described model. The results are presented in Figure 3. In the DQW quasidoublet spectrum, the spectral distribution appears symmetric relative to the $\langle x\rangle=0$ axis, due to relation (4). Furthermore, the energy spectrum of single electron can be sorted according to electron localization probability, as a set of localized states (in the left QW or right QW, for which $\langle x\rangle= \pm|\langle x\rangle|$ ), delocalized states (i.e., $\langle x\rangle \sim 0$ ), and states with different probabilities in left and right QWs. Competition between values of $\Delta E_{12}$ and the coupling coefficient $W$ in (2)-(3) defines the type of electron localization in the system. For

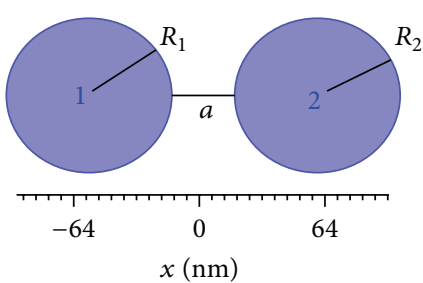

(a)

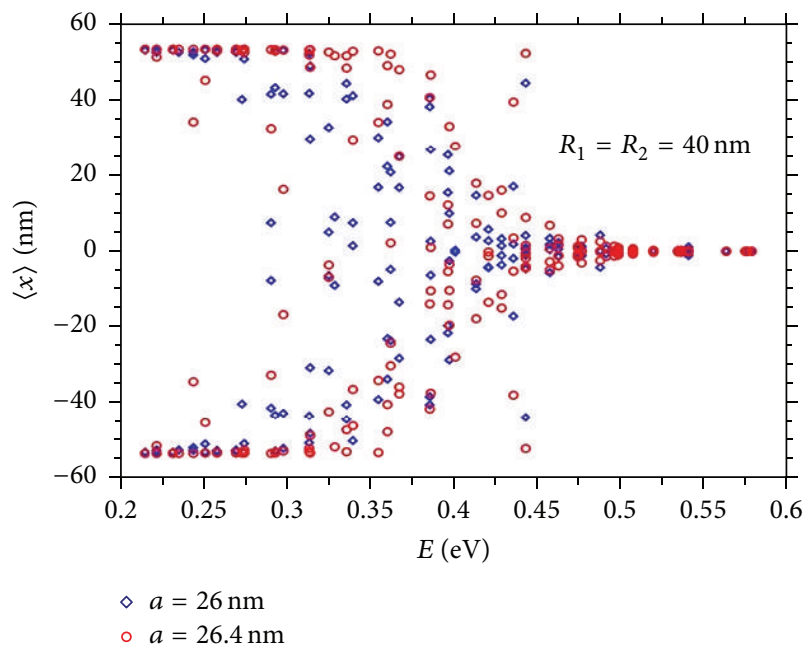

(b)

FIGURE 3: Effects of the interdot distance, $a$, on the spectrum of a DQW made of symmetric circular InAs/GaAs dots $\left(R_{1}=R_{2}=\right.$ $40 \mathrm{~nm}$ ). (a) Schematic representation of geometry parameters of DQW. (b) The average coordinate $\langle x\rangle$ calculated for each level of single electron DQW spectrum.

the ideal case of identical QWs, that is, when $\Delta E_{12}=0$, the wave functions of delocalized states are expressed as $\Psi_{+}=$ $\left(\psi_{1}+\psi_{2}\right) / \sqrt{2}$, and $\Psi_{-}=\left(-\psi_{1}+\psi_{2}\right) / \sqrt{2}$. It should be noted that numerical discretization of the Schrodinger equation on a finite mesh leads to significant computational errors. Thus the ideally symmetric system cannot be solved numerically, and computer modeling rather leads to solutions pertaining to dissymmetric QWs. As can be seen in Figure 3, there are strong deviations of the spectral distribution for the average coordinate $\langle x\rangle$, calculated for two interdot distances which differ for small values of $a$. This instability is stronger for the intermediate region of the spectrum, where the levels with different probability for localization in left and right QWs are found.

To evaluate the accuracy of the calculations we applied different meshes. Figure 4 shows the sensitivity of spectral distribution of $\langle x\rangle$ to tuning the discretization meshes. The results are unstable relative to mesh variations. In particular, the calculation for states with different localization probability (in left and right QWs) is unstable due to the uncertainty of $\Delta E_{12}$ (artificially due to computational errors) and relation (3). One can use the quasidoublet splitting energy $\Delta E=$ $E_{+}-E_{-}$at the ground state, as a reference, to evaluate the effect of discretization. Figure 4 shows $\langle x\rangle$ for three meshes and the inset gives the resulting $\Delta E$, energy splitting of the 


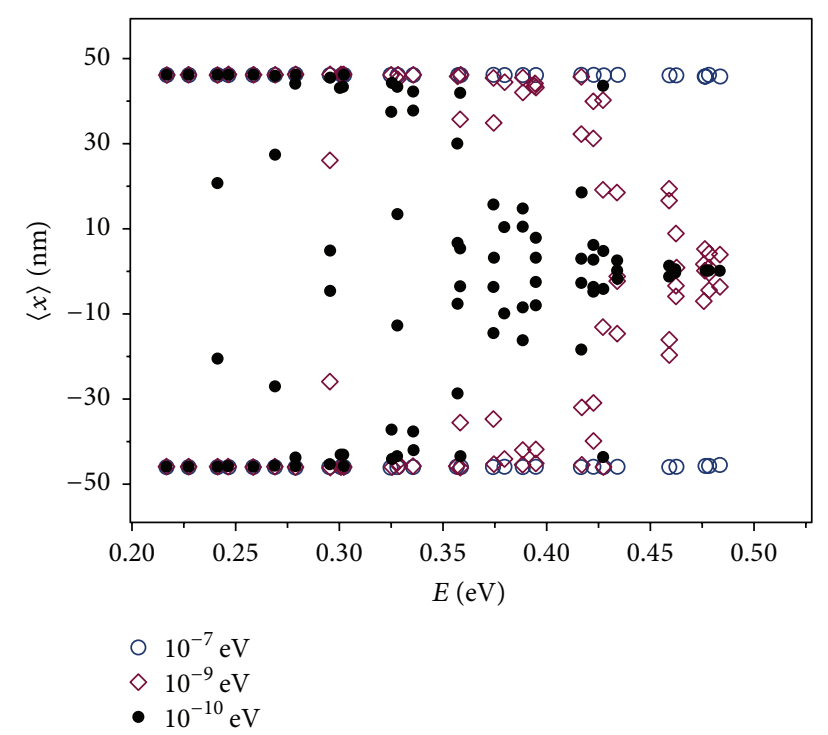

FIGURE 4: Spectral distributions for the average coordinate $\langle x\rangle$ for a circular DQW. The symbols correspond to discretization of the Schrödinger equation on various numerical meshes. The meshes differ by the elements minimum size. The energy splitting $(\Delta E)$ of the quasidoublet of the ground state for each discretization of a mesh is given in the inset. The finer mesh corresponds to smaller $\Delta E$. Interdot distance $a$ is kept the same and equal to $26 \mathrm{~nm}$.

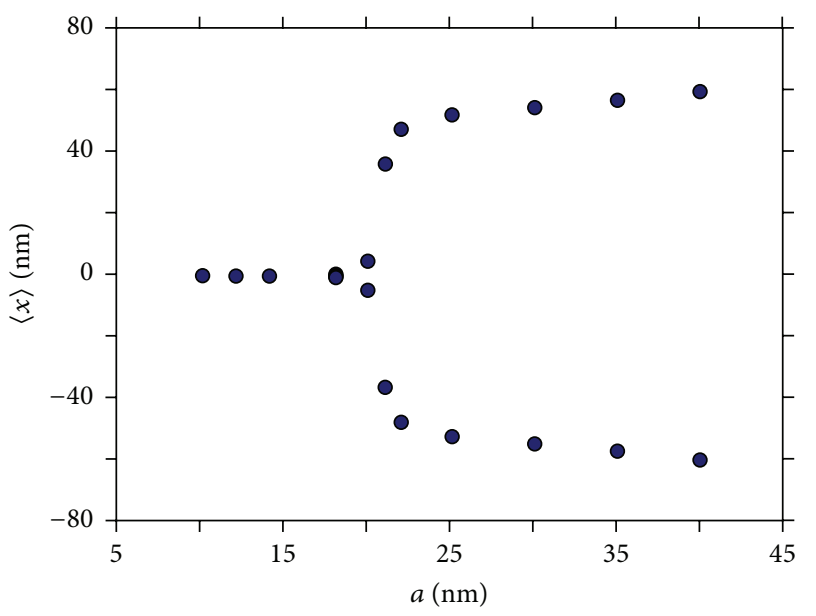

FIGURE 5: Average coordinate $\langle x\rangle$ of the electron in ground state of symmetric circle shaped DQW $\left(R_{1}=R_{2}=40 \mathrm{~nm}\right)$ as a function of the interdot distance. The numerical discretization for the used mesh leads to an energy difference $\Delta E \sim 10^{-7} \mathrm{eV}$ of the quasidoublet ground state.

quasidoublet for the ground state. It appears that the finer mesh gives the smaller $\Delta E$. Hence, we assume that the results of calculations presented below for identical QW have a numerical error of about $10^{-7} \mathrm{eV}$.

Visualization of the localized-delocalized states dynamics in the InAs/GaAs DQW is given in Figure 5, where the average coordinate $\langle x\rangle$ is presented as a function of the interdot distance $a$ (see also [7]). When the distance between QWs is large, the electron is localized in one of the QWs; in

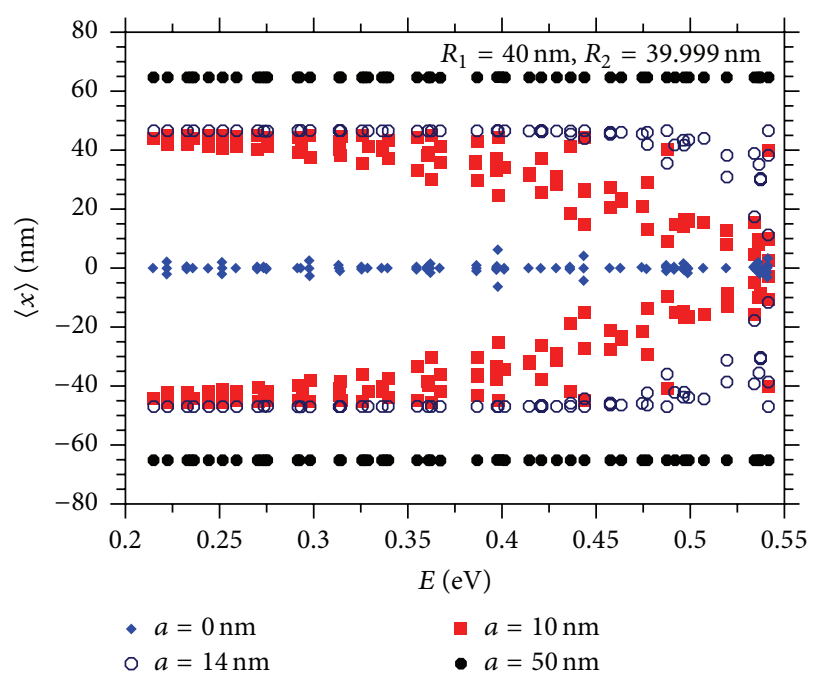

FIgURE 6: Average coordinate, $\langle x\rangle$, for single electron spectrum in the case of asymmetric DQW versus interdot distance $a$. The asymmetry parameter is maintained constant and equal to $\xi=$ $0.99975\left(\xi=R_{2} / R_{1}=39.999 / 40\right)$.

that case the parameter $\Theta \approx 0$. When the interdot distance decreases, the electron tunneling increases and the wave function spreads over the whole double system, $0 \leq \Theta \leq \pi$. All states become delocalized for interdot distances less than $17 \mathrm{~nm}$. The localized states occur for interdot distances larger than $25 \mathrm{~nm}$.

Additionally, as can be seen in (5), the electron localization demonstrates extreme sensitivity to small variations of QW shapes, which also violate the DQW left-right symmetry. Dissymmetry of dot shape will be discussed in the following section.

4.2. Asymmetric $D Q W$. Let us consider two circular QWs that are nonidentical. The asymmetry parameter for the DQW is defined as $\xi=R_{2} / R_{1}$. We chose very small asymmetry (e.g., $\xi \sim 0.999975=39.999 / 40$ ), as nearly identical QWs lead to relevant quantum effects and mimic the reality of QW arrays. In this study, we vary the interdot distance. The calculation results are presented in Figure 6, where the average coordinate $\langle x\rangle$ along the single electron spectrum is shown for four interdot distances. The interdot distance is gradually decreased from large distances, when the QWs are separated, to contacting QWs.

Comparing with the previously discussed identical DQWs, for asymmetric DQW, the interdot distance that causes the quantum states to become delocalized, over the entire spectrum, is smaller. For example, when $\xi=0.999975$, such interdot distance is less than $7 \mathrm{~nm}$, whereas it is equal to $17 \mathrm{~nm}$ for identical QWs.

It has been previously found [7] that extremely small difference in QW radii in a DQW (ratio $\xi=R_{2} / R_{1}$ slightly smaller than 1) drastically affects the spectral distribution of localized/delocalized states. Such violation of DQW shape symmetry defines small variation of $\Delta E_{12}$, according to (3). Also, according to (4), small change of $\Delta E_{12}$ can induce 


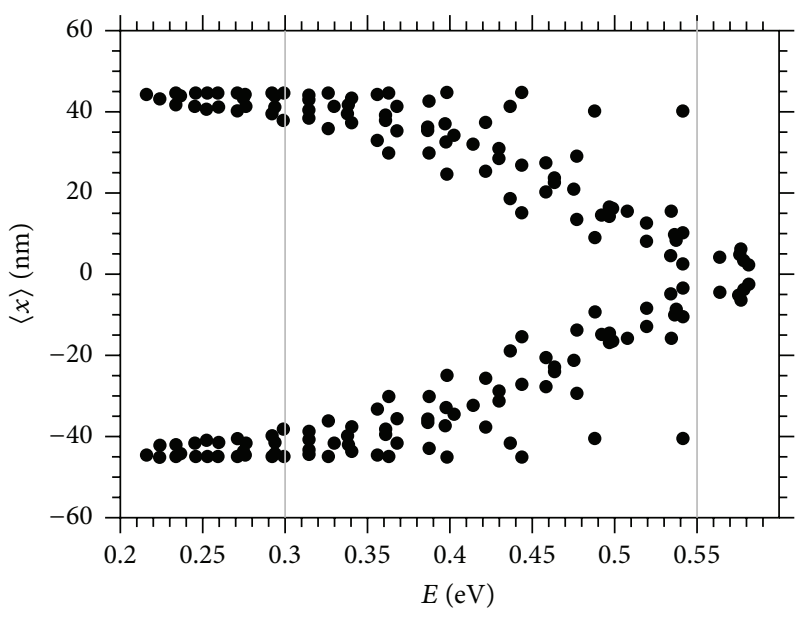

(a)

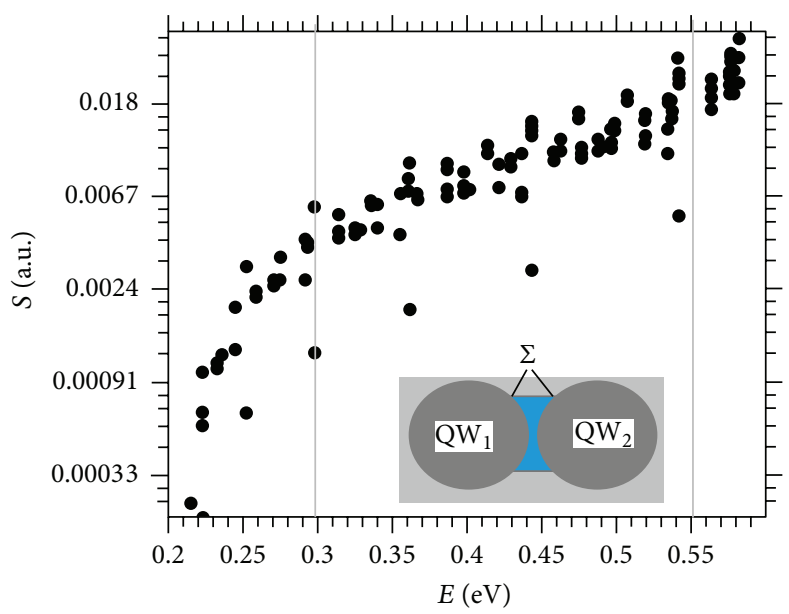

(b)

FIGURE 7: (a) Spectral distribution of $\langle x\rangle$ for asymmetric DQW with $a=10 \mathrm{~nm}$. The asymmetry parameter $\xi=R_{2} / R_{1}=39.999 / 40(\xi=$ 0.999975). The vertical lines divide the spectrum in three predominant parts: delocalized, transitional, and localized states. (b) Overlap integral $S$ plotted along electron spectrum; the integration area $\Sigma$ is shown in the inset.

significant changes of the coefficients of linear combination (2), thereby drastically changing the wave functions.

Typical picture for the spectral distribution of average coordinate $\langle x\rangle$ is shown in Figure 7(a), which was obtained for interdot distance $a=10 \mathrm{~nm}$. The spectrum readily divided in localized states (upper spectral levels), states with different localization probability in left and right QW (transitional states), and delocalized states (low-lying states). The relations in (4) explain the spectral distribution of the average coordinate shown in Figure 7(a).

The matrix element $W$ (which is also proportional to the quasidoublet energy splitting $\Delta E$ [18]) can be described using the following relation: $W \sim S$, where $S$ is the overlap integral, approximated by $S=\int_{\Sigma} \psi^{2}(x, y) d x d y$, with the integration domain $\Sigma$ being the area between the QWs; see inset in Figure 7(b). In the overlap integral, the wave functions $\psi(x, y)$ are normalized. The distribution of $S$ along the spectrum is shown in Figure 7(b). The magnitude of the wave function overlap depends on both the distance between quantum objects and the spreading of the wave function over the QWs. Generally, the matrix elements $W$ are larger for upper levels in comparison with those for low-lying levels. This is due to the fact that the upper levels of the spectrum have broader wave functions. The large values of $W$ are associated with the large $\Theta$ values (i.e., $\Theta \leq \pi$ ) for such levels; in such cases, the electron takes a localized state, as can be seen in Figure 7(a).

The symmetry breaking in DQW can be also made by variations of the confinement strength in one of the QWs. In Figure 8, the results of such variations are presented for QWs with identical shape. The magnitude of the DQW asymmetry is increased by increasing the potential from $V_{s}=0.21 \mathrm{eV}$ to $0.21005 \mathrm{eV}$, to $0.2101 \mathrm{eV}$, and to $0.211 \mathrm{eV}$. With the last value of $V_{s}$ all states in the spectrum become localized. Note that initially the states were delocalized, due to the chosen short interdot distance $(a=4 \mathrm{~nm})$. Again we see

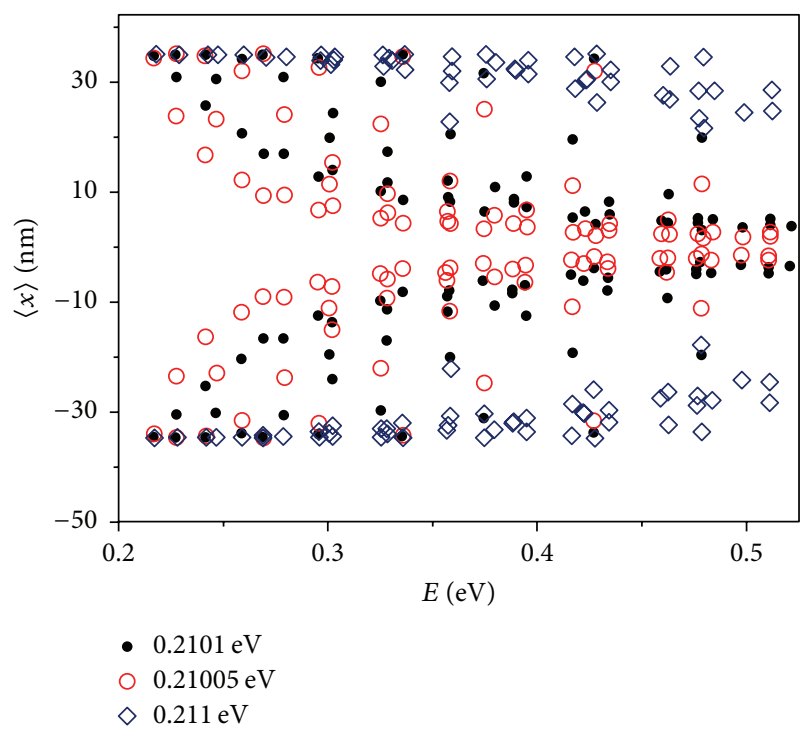

FIGURE 8: Single electron average coordinate $\langle x\rangle$ along the energy spectrum for asymmetric DQW under different confinement potentials $V_{s}$ in left QW (shown in the inset). The potential in right QW is not changed $\left(V_{s}=0.21 \mathrm{eV}\right)$. The interdot distance is kept constant and equal to $4 \mathrm{~nm}$.

that such quick transformation of electron localization (due to symmetry breaking) is possible under the condition of minimal uncertainty of electron energy. The pace and the sensitivity of the transformation are high for almost identical QWs.

One more illustration of symmetry breaking effect is given in Figure 9, where the DQW asymmetry is made by a small cut of the right quantum well $\left(\mathrm{QW}_{2}\right)$. The average coordinate $\langle x\rangle$ plotted along electron spectrum for different depths of the cut $\left(d_{0}=0.5 \mathrm{~nm}\right.$ and $\left.1 \mathrm{~nm}\right)$ is given in 


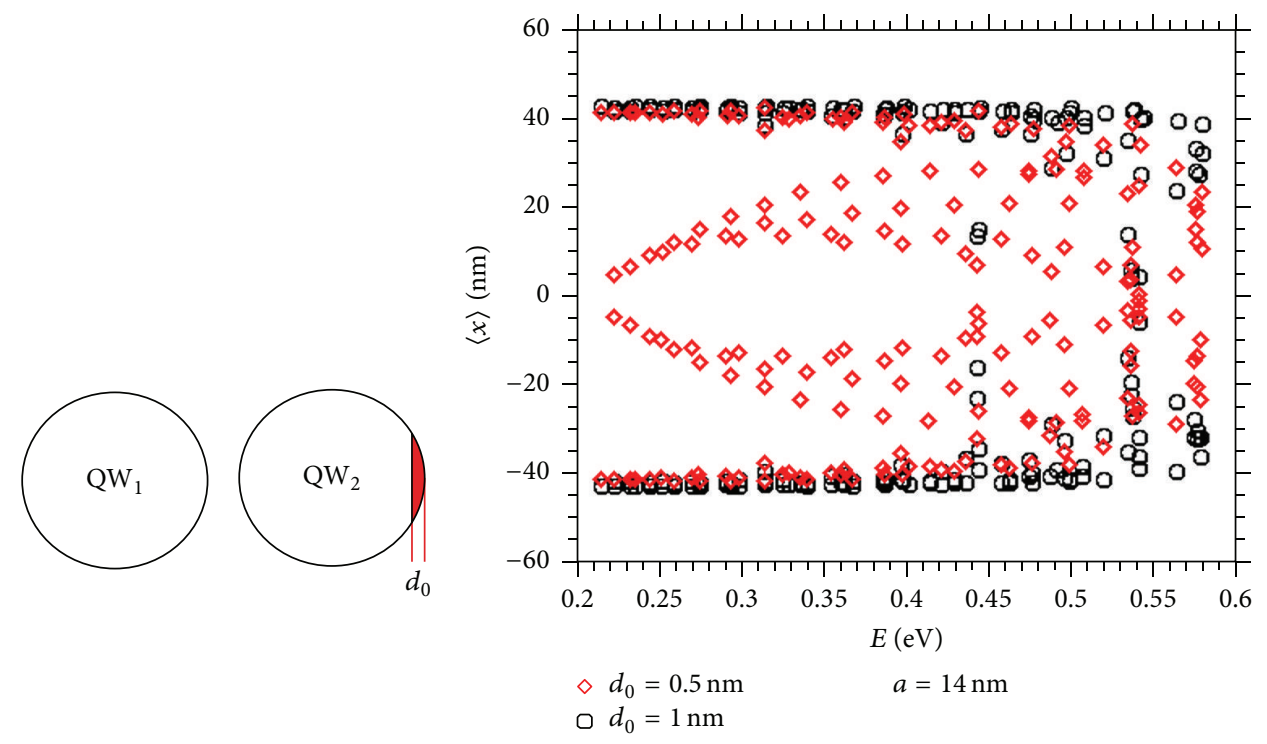

(a)

(b)

FIGURE 9: (a) Circle shaped DQW exhibiting an asymmetry generated by truncating the right quantum well $\left(\mathrm{QW}_{2}\right)$. (b) Electron average position $\langle x\rangle$ plotted along electron spectrum of the asymmetric DQWs $\left(R_{1}=R_{2}=40 \mathrm{~nm}\right.$ and $\left.a=14 \mathrm{~nm}\right)$ for two different cut depths $d_{0}$, inducing well contrasted symmetry violation within the DQW.

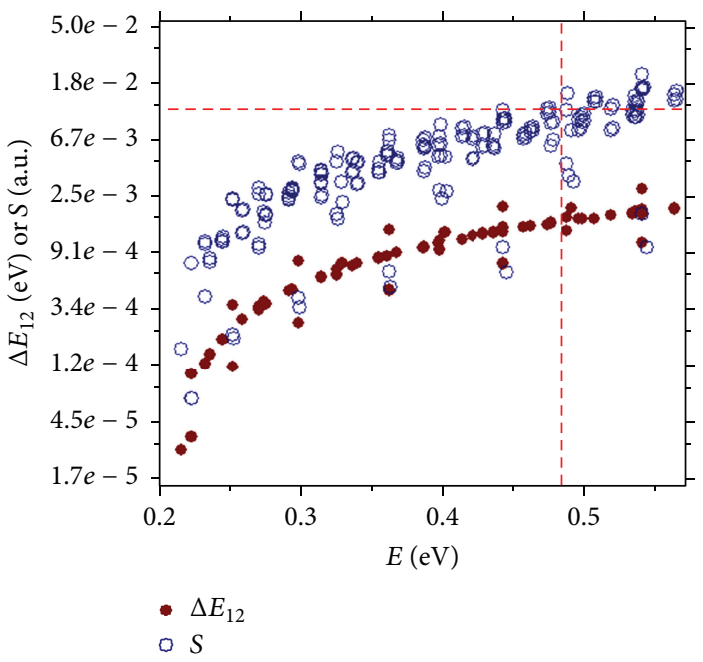

(a)

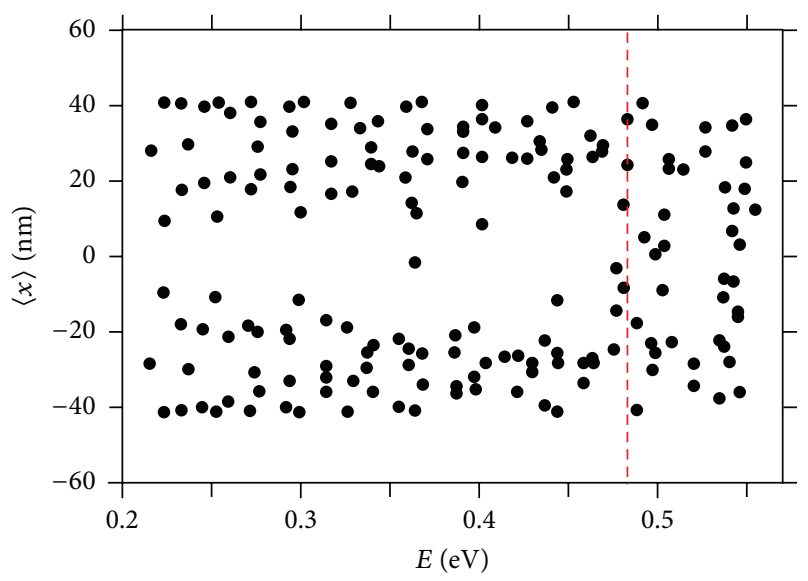

(b)

FIGURE 10: (a) Energy differences $\Delta E_{12}$ (solid circles) and overlap integrals $S$ (open circles). (b) Average coordinate $\langle x\rangle$ along single electron spectrum for the asymmetric DQW for asymmetry parameter $\xi=R_{2 y} / R_{2 x}, \xi=39.75 / 40=0.99375$. The dashed lines divide $\Delta E_{12}$ and $S$ ranges in regions of mainly localized and mainly delocalized states.

Figure 9(b). The interpretation of this data can be done by noticing that the decrease of the asymmetry (through shape variation or through decrease of energy uncertainty limits) leads to increasing uncertainty of electron position in the system. For larger depth of the cut, the states of the DQW are mainly localized states.

An additional illustration of the symmetry breaking effect is presented in Figures 10 and 11, where we compare the tunneling for two cases: small $(\xi=0.99375)$ and relatively large $(\xi=0.875)$ asymmetry of the DQW with fixed interdot distance. This is composed of a QW circular in shape with a radius $R_{1}=40 \mathrm{~nm}$ and an elliptical QW with the major axis along the $x$-axis $\left(R_{2 y} \leq R_{2 x}\right)$. The values $\Delta E_{12}$ and $S$ are shown in Figure 10(a). Again, the number of the delocalized levels appears larger for the case of small asymmetry. The "random" distribution of delocalized states in the spectrum relates to the distribution of the matrix element $W$ is reflected here in the distribution of the $S$ integral. The $S$ parameter varies up to two degrees for different spectral levels. The levels with minimal $S$ directly relate to delocalized states of the spectrum, as visible in Figure 10(b). One can note that if the value of $S$ is relatively large and $\Delta E_{12}$ is relatively small, the corresponding state 


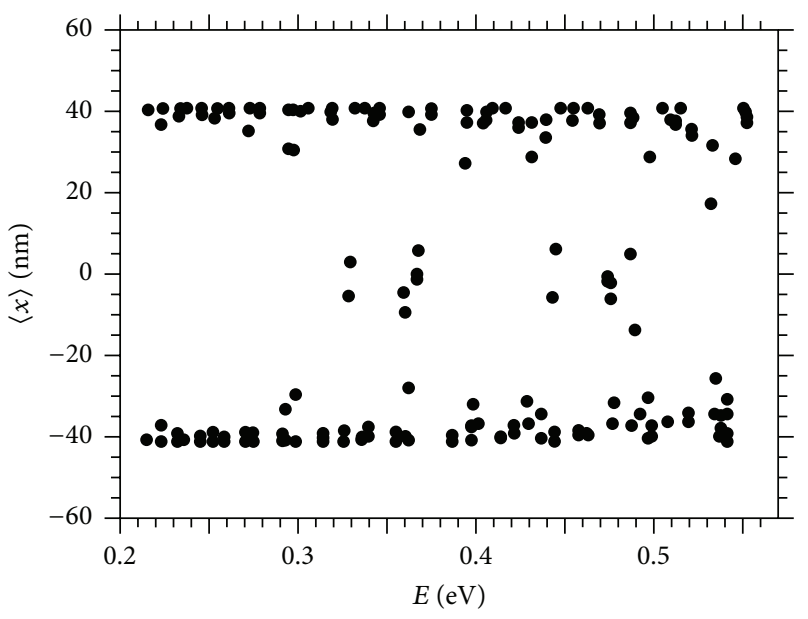

FIGURE 11: Average coordinate $\langle x\rangle$ along single electron spectrum for the asymmetric circular/elliptic DQW for asymmetry parameter $\xi=R_{2 y} / R_{2 x}, \xi=39.8 / 40=0.995$.

can be delocalized. Hence, we can divide the ranges of $\Delta E_{12}$ and $S$ in mainly localized states and mainly delocalized states regions (see vertical and horizontal lines). The number of delocalized states decreases when the asymmetry increases; these can be evaluated by comparing Figures $10(\mathrm{~b})$ and 11. It is clear that when the asymmetry becomes increasingly larger, the states with $\langle x\rangle \sim 0$ disappear.

The violation of DQW shape symmetry largely effects the spectral distribution of localized/delocalized states by varying the energy level splitting, manifested by a large energy difference $\Delta E_{12}$. This effect can favorably be applied to make a nanosensing functionality. We present in Figure 12 results of modeling an asymmetric DQW with a protuberance at the central edge of $\mathrm{QW}_{2}$, as is shown in Figure 12(a). The protuberance plays the role of either a defect grown with the quantum dot or a foreign material adsorbed on the surface of a dot that was initially identical to its peer.

It appears from data in Figure 12 that the degree of delocalization is a direct function of the size of the protuberance, which as indicated above could be a defect or an adsorbed substance. This offers the possibility to sense the protuberance size. Calibration of this sensing functionality can be done through our modeling and by varying the size of the defect, respectively, the adsorbed substance.

\section{Conclusion}

We studied the spectral distribution of localized/delocalized states in DQW. The electron localization in DQW appeared extremely sensitive to small violations of symmetry in DQWs due to relation (5) for $\Delta E_{12}$ nearing zero. The extreme sensitivity to symmetry violations, found through our modeling, can be technologically important.

The observed electron behavior in double quantum structures can be interpreted in terms of the uncertainty principle. We have shown that the spectral distribution of the electron average coordinate $\langle x\rangle$ is distinctly different for various types

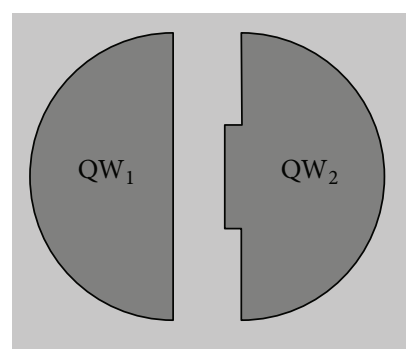

(a)

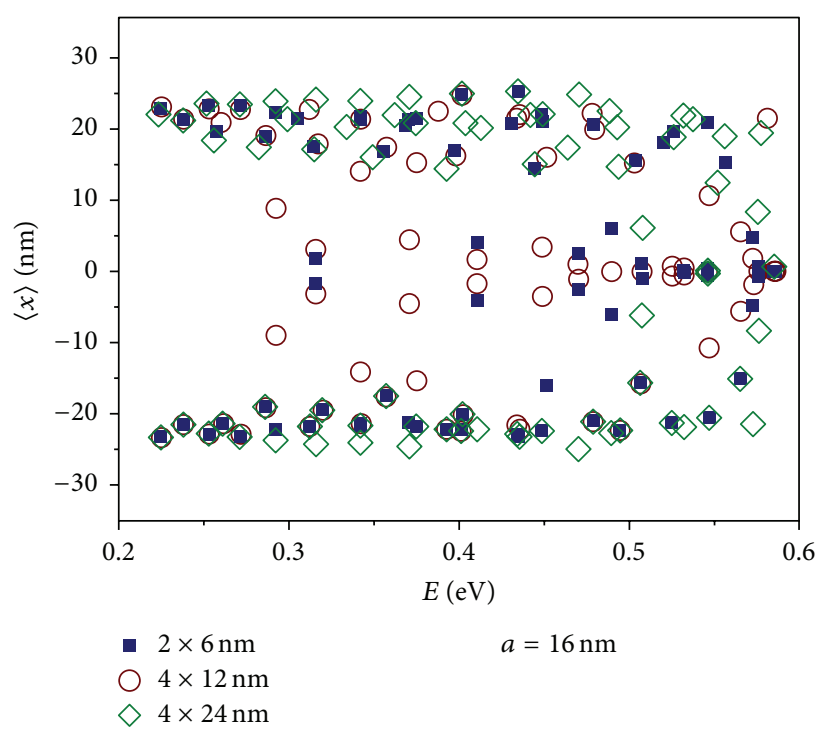

(b)

FIGURE 12: (a) Semicircle shaped DQW, where the asymmetry is generated by a rectangular defect in the right QW. The defect height, $H$, and width, $D$, were varied, as indicated in the inset, while the radii and the dot interdistance are maintained constant $\left(R_{1}=R_{2}=40 \mathrm{~nm}\right.$ and $a=16 \mathrm{~nm}$ ). (b) Electron average position $\langle x\rangle$ plotted against the electron spectrum of the asymmetric DQWs.

of DQW symmetry violations. It appeared that the ideal case of identical QWs cannot be reliably modeled numerically, since the closer the DQW to perfectly symmetric shape, the higher the effect of numerical discretization on tunneling properties.

We demonstrated that the DQW is a quantum system suitable for sensing tiny substances adsorbed on one of the quantum dots constituting the DQW or by a defect in one of the dots. The protuberance volume capable of breaking the symmetry, changing the quantum states of the DQW, and provoking tunneling was estimated to contain one to several thousand atoms, which is in the range of the size of polymer macromolecules and biomolecules.

\section{Conflict of Interests}

The authors declare that there is no conflict of interests regarding the publication of this paper. 


\section{Acknowledgments}

This work is supported by NSF (HRD-1345219) and MSRDC consortium (Award 001 W911SR-14-2-0001-0002).

\section{References}

[1] C. Cohen-Tannoudji, B. Diu, and F. Laloe, Quantum Mechanics, vol. 1, Wiley-VCH, Weinheim, Germany, 1977.

[2] L. R. C. Fonseca, J. L. Jimenez, and J. P. Leburton, "Anomalous peak effect in $\mathrm{CeRu}_{2}$ and $2 \mathrm{H}-\mathrm{NbSe}_{2}$ : fracturing of a flux line lattice," Physical Review B, vol. 58, no. 2, pp. 995-999, 1998.

[3] R. S. Whitney, H. Schomerus, and M. Kopp, "Semiclassical transport in nearly symmetric quantum dots. I. Symmetry breaking in the dot," Physical Review E, vol. 80, no. 5, Article ID 056209, 2009.

[4] Ł. Kłopotowski, M. Nawrocki, S. Maćkowski, and E. Janik, "Spin conserving tunneling in asymmetric double quantum well structures," Physica Status Solidi (B), vol. 229, no. 2, pp. 769-774, 2002.

[5] W. G. van der Wiel, S. De Franceschi, J. M. Elzerman, T. Fujisawa, S. Tarucha, and L. P. Kouwenhoven, "Electron transport through double quantum dots," Reviews of Modern Physics, vol. 75, no. 1, pp. 1-22, 2003.

[6] Y. I. Mazur, V. G. Dorogan, E. Marega Jr. et al., “Tunnelingbarrier controlled excitation transfer in hybrid quantum dotquantum well nanostructures," Journal of Applied Physics, vol. 108, no. 7, Article ID 074316, 2010.

[7] I. Filikhin, S. G. Matinyan, and B. Vlahovic, "Electron spectrum, localization and tunneling in double nanoscale structures," Mathematical Modelling and Geometry, vol. 2, no. 2, pp. 1-18, 2014.

[8] L. A. Ponomarenko, F. Schedin, M. I. Katsnelson et al., "Chaotic dirac billiard in graphene quantum dots," Science, vol. 320, no. 5874, pp. 356-358, 2008.

[9] R. S. Whitney, P. Marconcini, and M. Macucci, "Huge conductance peak caused by symmetry in double quantum dots," Physical Review Letters, vol. 102, no. 18, Article ID 186802, 2009.

[10] N. Ha, T. Mano, T. Kuroda et al., "Droplet epitaxy growth of telecom InAs quantum dots on metamorphic InAlAs/GaAs(111)A," Japanese Journal of Applied Physics, vol. 54, no. 4, supplement, Article ID 04DH07, 2015.

[11] N. Ha, X. Liu, T. Mano et al., "Droplet epitaxial growth of highly symmetric quantum dots emitting at telecommunication wavelengths on InP(111)A," Applied Physics Letters, vol. 104, no. 14, Article ID 143106, 2014.

[12] I. Filikhin, V. M. Suslov, and B. Vlahovic, "Modeling of In As/Ga As quantum ring capacitance spectroscopy in the nonparabolic approximation," Physical Review B, vol. 73, no. 20, Article ID 205332, 2006.

[13] I. Filikhin, V. M. Suslov, M. H. Wu, and B. Vlahovic, "Effective approach for strained InAs/GaAs quantum structures," Physica E: Low-Dimensional Systems and Nanostructures, vol. 40, no. 4, pp. 715-723, 2008.

[14] C. A. Duque, N. Porras-Montenegro, Z. Barticevic, M. Pacheco, and L. E. Oliveira, "Electron-hole transitions in self-assembled InAs/GaAs quantum dots: effects of applied magnetic fields and hydrostatic pressure," Microelectronics Journal, vol. 36, no. 3-6, pp. 231-233, 2005.

[15] M. Levinshtein, S. Rumyantsev, and M. Shur, Handbook Series on Semiconductor Parameters, World Scientific, Singapore, 1999.
[16] A. Schliwa, M. Winkelnkemper, and D. Bimberg, "Impact of size, shape, and composition on piezoelectric effects and electronic properties of In(Ga)As/GaAs quantum dots," Physical Review B, vol. 76, no. 20, Article ID 205324, 2007.

[17] I. Filikhin, V. M. Suslov, M. Wu, and B. Vlahovic, "InGaAs/GaAs quantum dots within an effective approach," Physica E: LowDimensional Systems and Nanostructures, vol. 41, no. 7, pp. 13581363, 2009.

[18] I. Filikhin, G. S. Matinyan, and B. Vlahovic, "Tunneling rate in double quantum wells," Sensors \& Transducers, vol. 183, no. 12, pp. 116-122, 2014. 

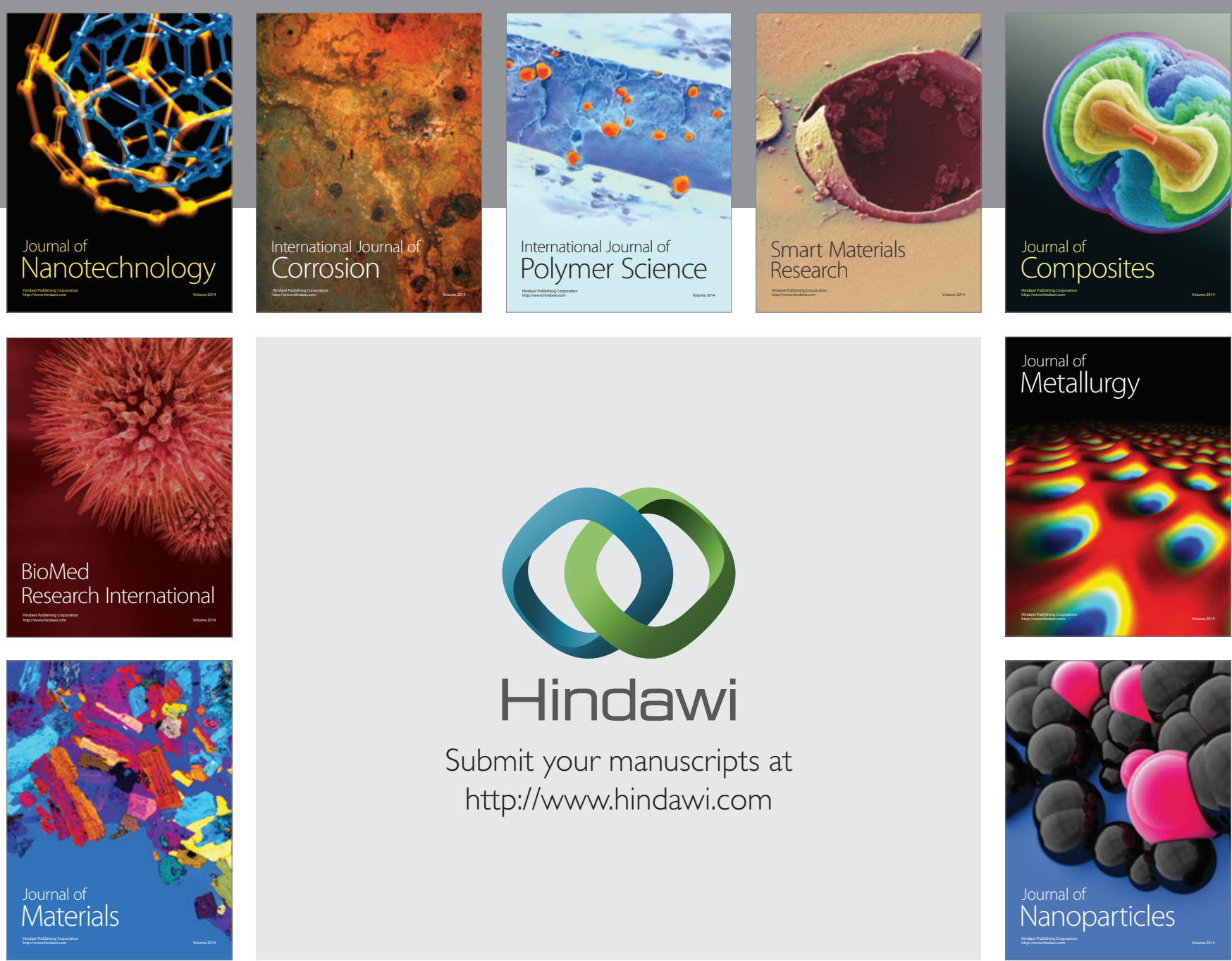

\section{Hindawi}

Submit your manuscripts at

http://www.hindawi.com

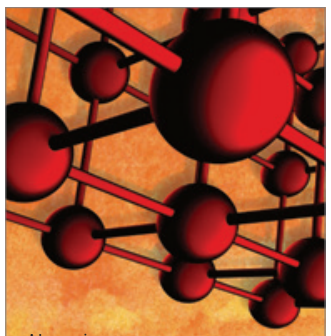

Materials Science and Engineering
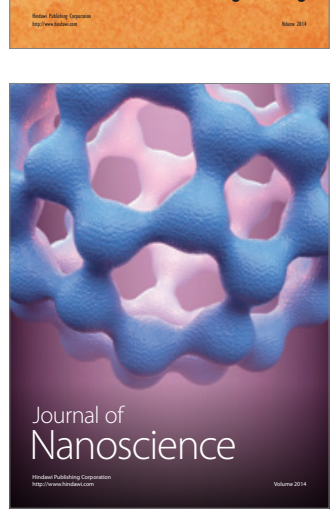
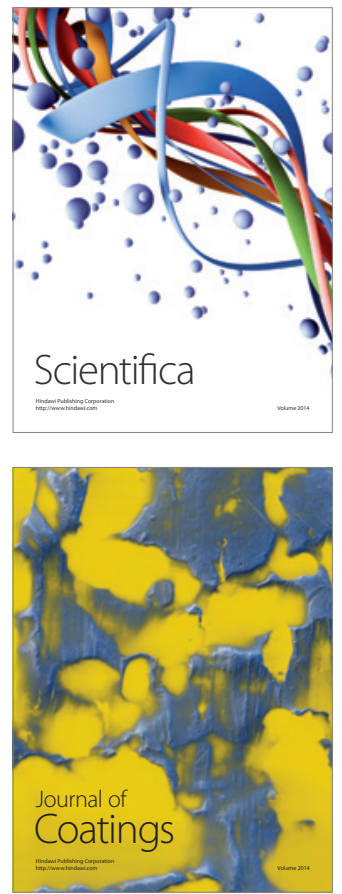
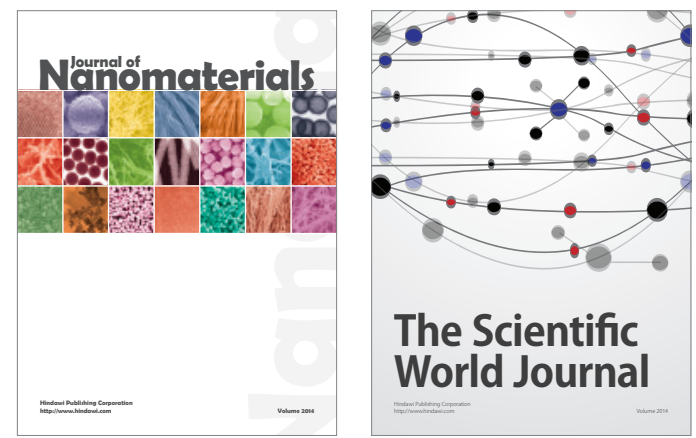

The Scientific World Journal
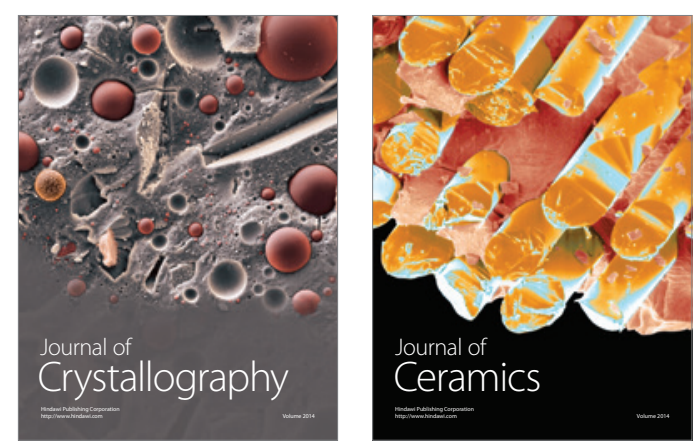
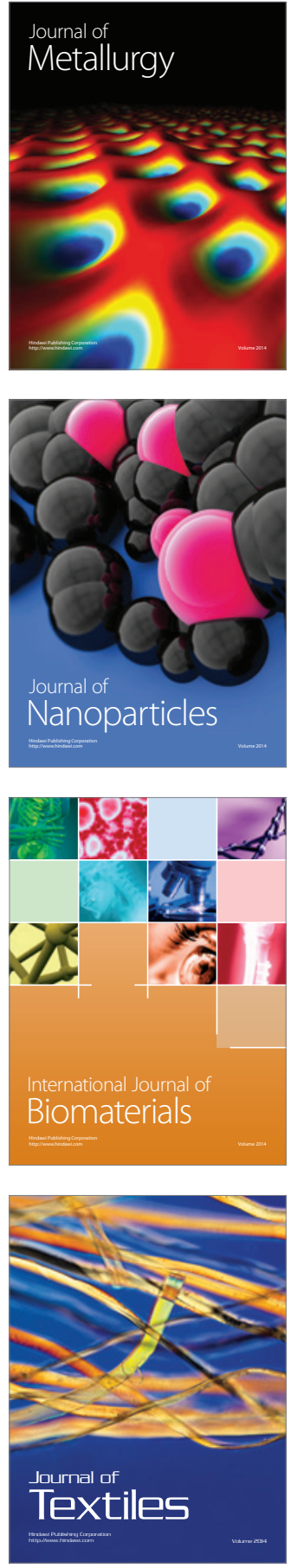